\title{
Velocity Distribution of the Protoplasmic Streaming in Nitella Cells*
}

\author{
by Noburô KAMIYA** and Kiyoko KURODA** \\ 神谷宣郎** ・黒田清子**：フラスモの細胞に批ける原形筫流動の速庋分布
}

Received June 30, 1956

One of the basic questions concerning the mechanism of protoplasmic streaming is whether the motive force of the flow is located within the streaming protoplasm itself or whether the driving energy is produced in the protoplasmic system not involved in streaming so that the moving protoplasm is driven passively. This is a point on which there is but little experimental evidence and there is a great divergence of opinions.

For an experimental approach to this problem it is necessary to determine how the velocity of streaming is distributed in the different layers of the flow, for without knowing and analysing it a sound discussion of the subject is scarcely possible. As early as 1903 Ewart paid attention to this problem and presented a figure of velocity distribution of protoplasm and cell sap in Chara cell which shows that the streaming is greatest in the inner layers of the endoplasm and least at the interface between endoplasm and ectoplasm; some degree of streaming is also shown by the cell sap in the layers adjacent to the vacuolar membrane. It was mainly these facts which led Ewart to believe that the energy for conducting streaming is developed in the endoplasm. His figure, however, does not appear to represent the situation of the intracellular motion exactly.

Since Ewart's report, there have been no experiments done along this line except Kamiya's work (1950) on the Myxomycete plasmodium. Our knowledge about the velocity distribution of the protoplasmic streaming in the cell having a cell wall is therefore as yet extremely meager. The purpose of the present paper is to throw light on the problem of the location of the motive force responsible for the streaming by investigating the intracellular velocity distribution of protoplasm exhibiting rota tion-one of the most orderly in pattern of various types of protoplasmic streaming.

In the following experiments we used cells of rhizoid, 'leaf', and internode of Nitella flexilis ${ }^{1)}$ as materials all of which show a vigorous and typical rotational streaming.

* Supported in part by the Grant in Aid for Fundamental Scientific Research from the Ministry of Education.

** Department of Biology, Faculty of Science, Osaka University, Osaka. 大阪大学理学部生物学教室

1) We used Nitella flexilis Agardh growing both in nature at Takarazuka near Osaka and in the Botanical Garden of Kyoto University. The material was identified by Professor K. Imahori of Kanazawa University, to whom the authors express here their appreciation. 


\section{Velocity Distribution of Protoplasm in a Rhizoid Cell}

The rhizoid cell is especially favourable for observing the velocity distribution due to its comparatively thick plasmasol layer (endoplasm) as well as due to the lack of chloroplasts, a fact which greatly facilitates the observation. The velocity of endoplasm at different layers can be determined by numerous minute granules with which the endoplasm is replete and which serve as index markers. As there are in the cell two streams occurring in the opposite directions, there exist also two indifferent zones between them where the flow stagnates. In order to determine the velocity of movement at different strata, we always placed the two indifferent zones in such a position that one was at the top and the other at the bottom of the cell, and observed the flow in the horizontal, optical section through the axis of the cell. By a simple microscopic observation we realize that all the endoplasmic layer moves together with a more or less equal rate along a very thin, almost imperceptible layer of cortical gej(ectoplasm). For measuring the velocity distribution at one and the same moment exactly, we resorted in this experiment to the cinematographic technique which was described by Kamiya(1950) when he applied it formerly for a similar purpose.

Fig. 1 represents one of the results thus obtained. Cytoplasmic granules found at a moment in an arbitrary transverse section $\mathrm{YY}^{\prime}$ were carried along with the stream to the positions shown by the open circles, the mean rate of the granules being $21 \mu / \mathrm{sec}$. This figure demonstrates that there is, if any,

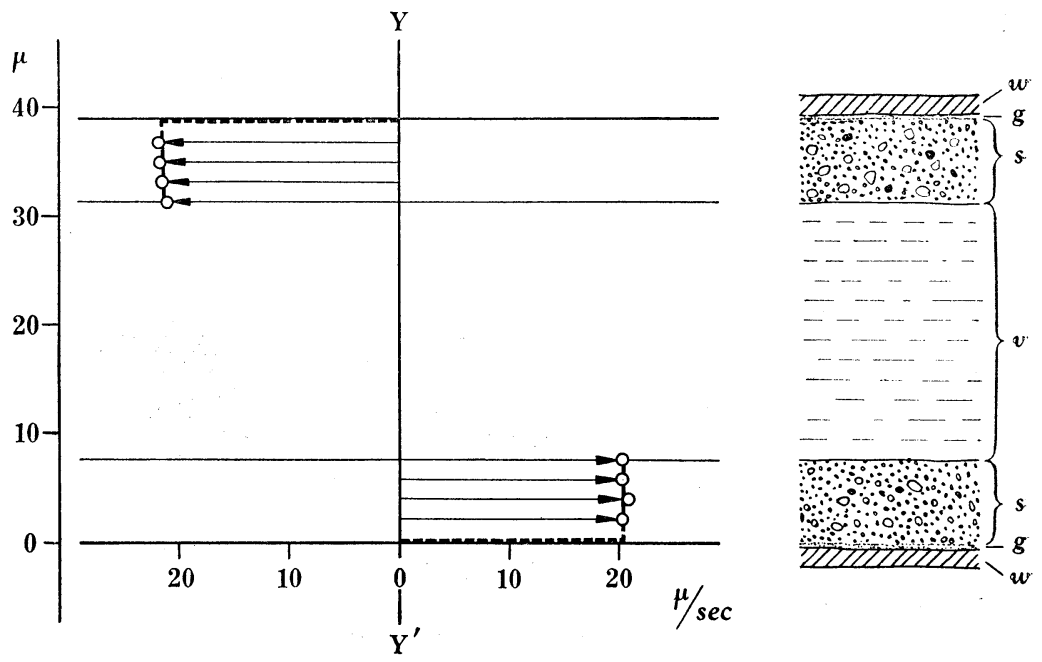

Fig. 1. Velocity distribution of the endoplasmic streaming in a rhizoid cell of Nitella flexilis. (Temp. $23^{\circ} \mathrm{C}$.) A part of the rhizoid cell as seen under the microscope is shown on the right. $w$ : cell wall, $g$ : plasmagel (ectoplasm), $s$ : plasmasol (endoplasm), $v$ : vacuole.

an only insignificant velocity gradient in the endoplasm itself. Only in the regions very close to the cortical gel layers, where the velocity is represented with broken lines, an extremely large velocity gradient is found. In other words, the plasmasol layer (endoplasm) is actually not streaming, but just slipping as a whole on the inner surface of the cortical layer. This is a fact which has not been duly expressed or emphasized before, but is extremely important in looking into the nature of proto- 
plasmic streaming.

We could not determine the velocity distribution of the cell sap in this case, as there were not enough suspended particles in the vacuole.

\section{Velocity Distribution of the Protoplasm and Cell Sap in a Young Leaf Cell}

As a young 'leaf' cell is rich in the amount of protoplasm taking part in the streaming and also in suspended inclusions in the cell sap, it permits us to determine the velocity distribution of both protoplasm and cell sap. Furthermore, the situation is simpler than the grown-up internodal cells in that the course of flow is not spirally wound but straight.

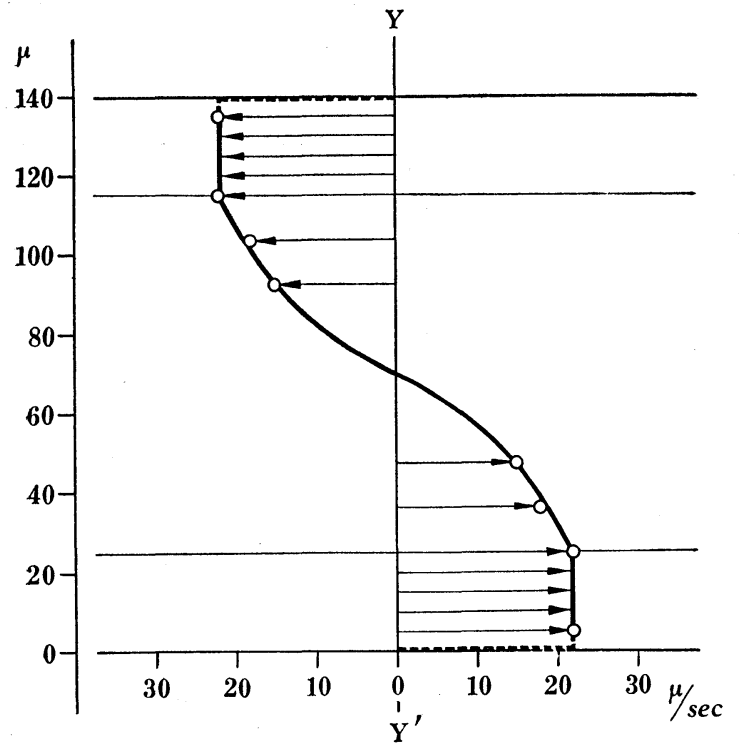

Fig. 2. Velocity distribution of the streaming of protoplasm and cell sap in a young 'leaf' cell. (Temp. $17^{\circ} \mathrm{C}$.) A part of the leaf cell as seen under the microscope is shown on the right. $w$ : cell wall, $g$ : plasmagel (ectoplasm), $s$ : plasmasol (endoplasm), $v:$ vacuole.

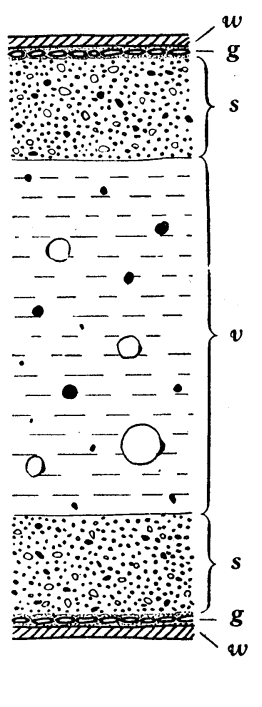

Fig. 2 shows the intracellular motion in a cell of this kind. The two indifferent zones between the two opposing streams are in this case, too, one at the top, and the other at the bottom of the cell, and the figure represents the velocity in the optical section through the central axis. From this figure we see that the rates of flow at

various endoplasmic layers are again the same just as in the case of rhizoid cell in Fig. 1 .

The velocity of the sap, which wets the tonoplast and is carried passively by it, decreases inwardly until the central axis is reached where the velocity is nil. On the other hand the velocity gradient of the sap, which is indicated by the slope of the curve against the transverse section of the cell, increases inwardly until the maximum is reached at the point of inflection found at the middle point of the vacuole. The velocity gradients of the cell sap in the peripheral and axial regions of the vacuole were $0.25 \mu / \mathrm{sec}$. and $1.0 \mu / \mathrm{sec}$. respectively per parallel strata $1 \mu$ apart. 


\section{Velocity Distribution of Protoplasm in a Cell Having no Vacuole}

It was reported by Hayashi (1952) that in a strangulated fragment of an internodal cell, the entire space of which is filled with the endoplasm, the protoplasmic streaming still continues, though greatly restricted.

A cell fragment completely filled

with protoplasm was made artificially by combining centrifugation and subsequent ligation of the cell (Fig. 3). When an internodal cell (Fig. $3 a$ ) is centrifuged gently (1200-1500 r.p.m.) for 10 minutes, the greater part of the endoplasm is forced to the centrifugal end leaving the cortical gel layer behind together with the chloroplasts imbedded in it(Fig. $3 b$ ). The amount of plasmasol (endoplasm) taking part in streaming, which may be estimated by this method, was shown in our material to be about $1 / 5-1 / 7$ of the whole cell volume. If the cell is left untouched, the endoplasm thus dislocated to one end of the cell soon begins to flow back spontaneously along the same course in the same direction as before. In case the centrifugal treatment is strong enough

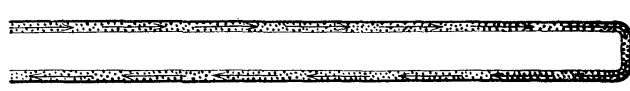

(a)
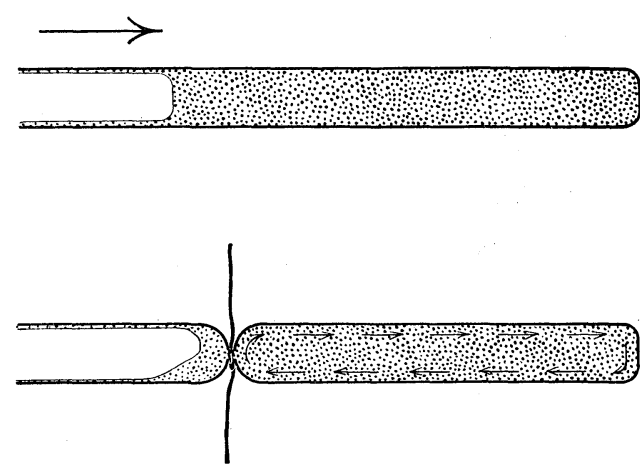

Fig. 3. The procedure for obtaining a plasmfilled cell fragment, represented diagrammatically. a) One end of an internodal cell of Nitella under natural condition. Chloroplasts in the cortical layer are not shown. b) One end of the cell at which the endoplasm is accumulated under the effect of centrifugal force. Arrow indicates the direction of centrifugation. c) A cell fragment produced by strangulation from the centrifuged cell.

as to tear off the cortical gel or to injure its structure, the cell ceases to survive. So far as the cortical gel layer remains intact, however, the forced accumulation of endoplasm at one end of the cell is by no means fatal.

If we tie off such a centrifuged internodal cell promptly with a strip of silk thread near the centrifugal end of the cell before the endoplasm flows back, a cell fragment as long as $10 \mathrm{~mm}$ completely filled with plasmasol can be obtained (Fig. $3 c$ ). The strangulated cell fragment filled with endoplasm in this manner can grow, if cultured properly, new vacuoles appearing sooner or later (Hayashi, 1952). As a tied-off fragment of the internodal cell keeps all the functions that an intact cell has, we shall hereafter call it simply a cell. Strangulation of Nitella-cell has been used recently for elucidating various cell-physiological problems (Sandan, 1955; Kamiya and Kuroda, 1956; Kuroda, 1956).

The cell which is completety filled with endoplasm and has no visible vacuole in 
itself showed a rather vigorous streaming in our experiment. This is a fact which shows undoubtedly that the existence of the vacuole is not a sine qua non condition for the flowing of the protoplasm. Our purpose now is to determine how the velocity of the protoplasmic streaming is distributed in such a cell.

An example

of the velocity distribution is represented in Fig. 4. The cell from which Fig. 4 was drawn was $7.5 \mathrm{~mm}$ in length and $0.46 \mathrm{~mm}$ in width. The measurement of the rate of flow at different strata was done in this case successively at different time using a stopwatch and an ocular micrometer. However, the flow
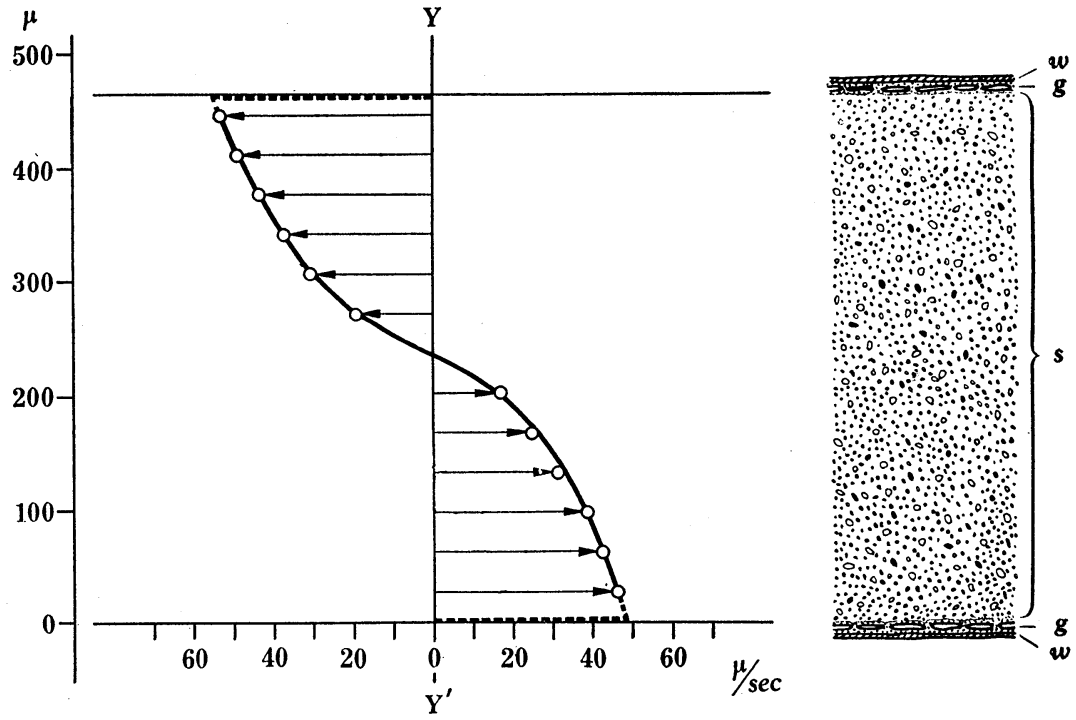

Fig. 4. Velocity distribution of the endoplasmic streaming in a cell, the entire space of which is filled artificially with endoplasm. (Temp. $28^{\circ} \mathrm{C}$.) A part of the cell as seen under the microscope is shown on the right. $w$ : cell wall, $g$ : plasmagel (ectoplasm) in which chloroplasts are imbedded. $s$ : plasmasol (endoplasm). No vacuole is visible. The rate of protoplasmic streaming in an intact internodal cell was $78 \mu / \mathrm{sec}$. at $28^{\circ} \mathrm{C}$.

being stable and stationary, we can figure out from the values thus obtained the velocity distribution of the endoplasmic streaming in respect to one and the same moment. The figure represents the velocity of flow in the optical section through the axis of the cell.

It is noticed here that the streams in the two halves of the cell space divided by the central plane connecting the two indifferent striations go in the opposite directions. The two indifferent striations, which take gentle spiral courses $\left(9^{\circ}\right.$ against the longitudinal axis) in this case, pass, one on the top, and the other at the bottom of the cell in the transverse section $Y^{\prime}$. In the optical section, in which the velocity at different strata was measured, the endoplasm flows either upward or downward one or the other side making an angle of $9^{\circ}$ with the optical section. Therefore the actual velocity must be the value of the measured velocity multiplied by $1 / \cos 9^{\circ}$. This is, however, regarded as being equal to the measured velocity itself, since $1 / \cos 9^{\circ}$ is 1.01 . The rate of flow is shown in the figure to be greatest in the peripheral layer and becomes gradually less as the central axis is approached. The inclination of the curve against the transverse plane $Y Y^{\prime}$ is stee- 
per in the central region of the cell than in the neighbourhood of the peripheries. Thus the curve takes a sigmoid shape with the point of inflexion at the axis of the cell. Here the velocity gradient of the endoplasmic flow in the peripheral region of the cell is $0.1 \mu / \mathrm{sec}$. and that in the middle region of the cell is $0.6 \mu / \mathrm{sec}$. per parallel strata $1 \mu$ apart.

In the layers adjacent to the interface between sol and gel, the curve is represented with broken lines as was also the case in the foregoing. This is because the velocity gradient at these regions is so great, that the exact determination of the shape of velocity distribution is impracticable. As shown in the figure, the rate of endoplasmic flow is greater the nearer the periphery is, but it appears that the greatest velocity near the periphery suddenly drops to zero as the cortical gel is reached. The velocity curve is therefore almost discontinuous at both ends near the peripheries.

All in all it is clear enough, as is also the case in Figs. 1 and 2 , that the velocity gradient is enormously high in the interfacial zone between the cortical gel layer and the outermost endoplasm. Not only that, but we also become aware that the sigmoid type velocity distribution of the endoplasm shown in Fig. 4 is very similar in form to that of the cell sap in Fig. 2. This is a point which has an important bearing to the mechanism of cyclosis and will be considered more fully in the fo!lowing.

\section{Model Experiment}

In order to demonstrate that the sigmoid type velocity distribution is a necessary consequence of the inner fluid flowing passively when two equal halves of the peripheral zone facing each other in a circular tube are shifted in parallel in the opposite directions, a simple model experiment was attempted instead of trying a tedious mathematical treatment. This kind of model experiment is straight-forward and illuminating in understanding the meaning of the shape of velocity distribution obtained above, and is sufficient for our present purpose.

For conducting the experiment, we prepared a circular, transparent tube made of methyl methacrylate having a bore of $19 \mathrm{~mm}$ which was cut longitudinally into equal halves having a semi-cylindrical wall. These walls were brought face to face nto a guiding frame so that a complete tube was formed. The construction of the frame is such that it is provided with two projected rails so that the upper and lower semi-cylindrical walls can slide smoothly along them (Fig. $5 b$ ).

As a substance to be filled in the tube we found margarine for kitchen use to be best suited for our purpose of observing how far the inner fluid material travels, when the two opposite walls slide against each other. In practice, the right half of the tube was filled with plain margarine and the left half with margarine that was stained red with carmine, and the whole setup was kept at $33^{\circ} \mathrm{C}$. so that the entire content became sufficiently fluid; the boundary between plain and red 


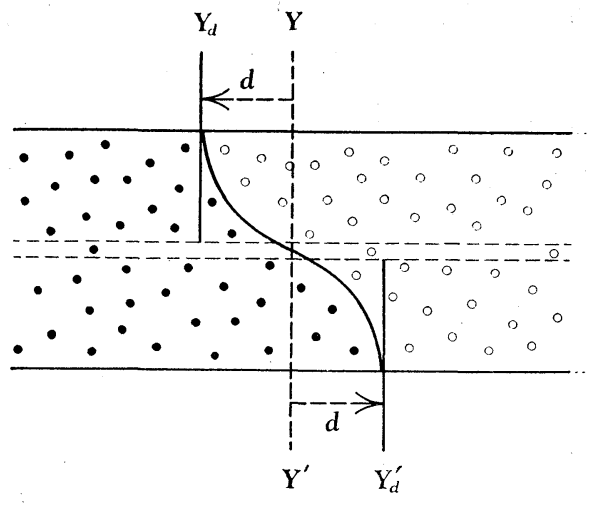

(a)

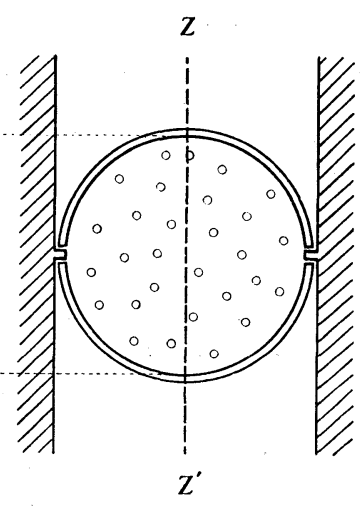

( b)
Fig. 5. Model experiment for showing the distribution of a fluid material in a tube when the two equal halves of the tube were made to shift longitudinally against each other. a) The longitudinal section of the tube after the upper and lower walls were moved. from $Y Y^{\prime}$ line to $Y_{d}$ and $Y^{\prime}{ }_{d}$ respectively. The space represented by solid circles is filled with margarine stained with carmine and that represented by open circles with plain margarine. $b$ ) The cross: section of the tube consisting of two sliding semi-cylindrical walls which are placed face to face in the guiding frame on two projecting rails. For further explanations, see text. margarine, however, must be left flat and perpendicular to the axis. The next step was to slide the two semi-cylindrical walls against each other in opposite directions for a definite distance, $d$, always keeping them tightly on the guid. ing rails of the frame. Then the setup filled with mar. garine was brought into a refrigerator and kept in it until their content got sufficiently hard. We are thus ready to

remove the walls and cut the cylindrical margarine longitudinally through the median plane $Z^{\prime}$ (Fig. $5 b$ ), which is perpendicular to the sliding plane. How this section looks like is shown in Fig. 5a. and Fig. 6.

The similarity of the shape of the boundary thus obtained in this model to the form of velocity distribution of the cell sap (Fig. 2) as well as to that of the protoplasmic flow in a plasmfilled cell (Fig. 4) is striking. The shape of these curves may of course not be exactly the same, because the rheological properties, as expressed by flow-pressure diagram (i.e., velocity gradient-shearing force diagram), of the endoplasm, cell sap, and warm liquid margarine may not be identical. Nevertheless, the fact that the protoplasm in a vacuole-free cell streams with an intracellular velocity distribution which has the same general pat-

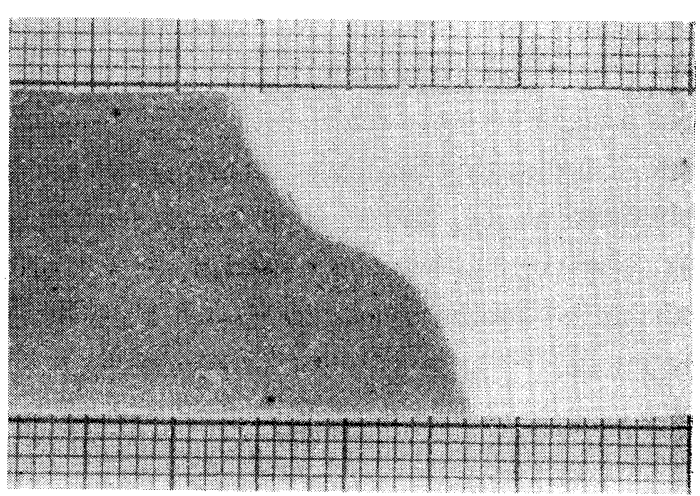

Fig. 6. Deformation of the boundary between red and yellow margarine after the upper and lower walls of the tube (removed before the section was made) were shifted against each other. This shows the longitudinal section through the median plane $Z Z^{\prime}$ shown in Fig. 5 b. The boundary was originally flat and perpendicular to the longitudinal axis. 
tern as that of the deformation curve obtained in the above experiment, or that of the velocity distribution of the cell sap, has a significant implication, namely that the endoplasm moves just like an inert liquid finding itself between two semicylindrical walls that are made to slide against each other in the longitudinal direction. This suggests that the endoplasmic flow in Fig. 4. is induced passively. What induces the endoplsm to stream, then, must be the force generated at the interface between plasmasol and plasmagel.

\section{Considerations}

It was shown in the foregoing observation that in a rhizoid cell as well as in a 'leaf' cell under natural state, the plamasol (endoplasm) at different layers moves with a nearly equal rate. In other words, there is only a negligible velocity gradient within the moving endoplasmic layer. The movement of endoplasm in one direction takes place along the ectoplasmic wall, the cross section of which is semicircular. The two borders existing between the opposing streams form indifferent zones, which are rather narrow. As the tonoplast is carried with the plasmasol, the direction of its movement across the vacuole is opposite to each other. The cell sap in the vacuole is therefore necessarily subjected to the shearing force between the opposing streams of endoplasm. Hence the situation in the cell sap in the vacuole is comparable, in respect to velocity gradient, to that of the fluid filled in a tube consisting of two sliding walls described above. No wonder that the velocity distribution of the cell sap (Fig. 2) and the shape of the boundary shown in Fig. 6 are very similar to each other.

It is, on the other hand, worth noticing, that the form of velocity distribution of the endoplasm shown in Fig. 4 is also identical in its basic character to that of the cell sap or of the fluid (warm margarine!) in the sliding wall system. This fact provides a ground for us to conjecture that the endoplasmic motion is also passively caused and the region which plays an active part in driving the endoplasm is located at the boundary between the cortical gel and the endoplasm where the velocity gradient is enormously high.

The fact that the velocity gradient is negligible within the flowing layer of endoplasm in a cell having a large central vacuole is to be understood as being due to the situation that the endoplasm is considerably higher in viscosity than the cell sap. Therefore the absence of the velocity gradient in this case is a further confirmation of the inference that the plasmasol (endoplasm) is inert and it alone can take no active part in the streaming.

Thus all the data obtained by us positively support the view that the active driving mechanism functions only at the boundary zone between plasmasol and plasmasgel. All other portions of the endoplasm, at least in the case of Nitella cell, appears to be passive in respect to movement.

The fact that, when the plasmagel layer is injured locally, the endoplasmic 
flow no longer occurs on the very spot but goes around it also shows that the special organization in the plasmagel layer is essential for maintaining the protoplasmic streaming (Linsbauer, 1929).

That the plasmasol alone is unable to flow actively is also to be shown by the experiment of isolated naked plasmasol drops from the internodal cell of Nitella. By means of a special method of amputation applying a negative hydrostatic pressure to the cell (Kamiya and Kuroda, unpublished), we obtained with least injury very large protoplasmic drops (more than $200 \mu$ in diameter) which can survive more than 50 hours in an artificial medium. What we should like to mention here about these isolated protoplasmic drops which came from the plasmasol part (endoplasm) of the cell, is that they no longer show any rotational streaming as was observed when it was in the cell. This is comprehensible if we consider it as being due to the fact that there is no organized cortical gel layer in these extracellular endoplasmic drops. It is, however, striking enough that a few chloroplasts, which were probably suspended freely in the endoplasm before the cell was cut, show a vigorous rotation each around its own axis. Such a revolving motion of the individual plastids continues usually several hours, sometimes even 2 days. Again, this phenomenon is interpreted neatly as representing the interfacial phenomena between sol and gel. Plasmagel in this case is freely suspended in the endoplasmic drop and consequently: what can occur as a result of shearing force acting at the surface of the gel particles (chloroplasts) is not the mass streaming of the sol phase but the individual revolving motion of the gel particles. As a matter of fact a minute, but rapid streaming in the opposite direction is perceptible by a careful observation in the immediate proximity of the surface of the rotating plastids as was also pointed out by Valkanov (1934) in connection with his obserbation of the revolving motion of the nucleus.

The above facts are further in conformity with the conclusion that the interaction of organized gel surface and sol phase produces the shearing force which brings about the interfacial slippage.

The generality of the conclusion obtained by us in Nitella-cells is naturally to e checked in other protoplasmic systems. In the case of Myxomycete plasmodium, Kamiya (1950) pointed out that the velocity distribution of protoplasm when it flows in the capillary tube of its own is extremely similar to that of a non-Newtonian liquid transpiring through a rigid circular tube. Furthermore, the endoplasmic flow caused solely artificially in the double-chambar under a pressure difference, gives also a figure of extrusion identical with that of the spontaneous natural flow. This fact undoubtedly indicates that the normal flow o protoplasm in the capillary tube of the plasmodium under natural condition is caused by a local difference in pressure established in the same protoplasmic system. The problem concerning he mechanism of the protoplasmic streaming in the slime mould is, herefore, substituted for the problem as to how a local difference in pressure is established in a proto- 
plasmic system. What comes into focus concerning the rotational movement in Nitella, on the other hand, is the mechanism causing the slippage between the solgel interface.

The velocity distribution in the form of a flat-headed parabola, as seen in the intracapillary flow of the endoplasm of the Myxomycete plasmodium (Kamiya, 1950), and the velocity distribution appearing in a sigmoid form, as seen in a plasm-filled Nitella cell in Fig. 4, are the same in nature in that they are both passive. Their forms are merely rendered different by the fact that in the former there is a pressure differenece between the anterior and the posterior parts of the tube whereas in the latter the endoplasm finds itself between two semicylindrical surfaces that are made to move in the opposite directions.

The generation of pressure difference occurring locally in a continuous protoplasmic system, as seen in a slime mould, and the generation of a force that brings about a slippage in the interface region between sol and gel layers, as seen in rotational streaming, both belong phenomenologically to the category of "protoplasmic streaming" in as much as they equally bring about streaming. It remains, however, a question how far they have a common basis in respect to their mechanism. It is expected that sometime in the future they will be included in one theoretical system. Though we are really seeking for such a unified picture of movement in protoplasm, it seems at present that there are not enough experimental support warranting such a speculation. It must be said that we are yet in a stage where we have to investigate each phenomenon separately without any preconceived ideas.

\section{Summary}

1) The intracellular velocity distribution of the rotational streaming was determined in cells of rhizoid, 'leaf' and internode of Nitella flexilis.

2) In the cells havig a central vacuole, the plasmasol (endoplasm) at different layers flows with a nearly equal rate giving rise to an only insignificant velocity gradient inside. Only in the very narrow interfacial region between sol and gel layers there is an enormous velocity gradient.

3) In the cell, the whole space of which is filled artificially with endoplasm, the streaming of endoplasm occurs with a velocity distribution which coincides with that we would expect the endoplasm to assume when it is moved passively between two semicylindrical walls which shift in the opposite directions.

4) On the basis of analysis of the intracellular velocity distribution with the aid of a model experiment it was concluded that the flow of endoplasm is passive; the mechanism which drives endoplasm actively resides in the interfacial region between plasmasol (endoplasm) and plasmagel (ectoplasm). 


\title{
References
}

Ewart, A. J., Physics and Physiology of Protoplasmic Streaming in Plants. Oxford (1903). Hayashi, T., Bot. Mag. Tokyo 65 : 51 (1952). Kamiya, N., Cytologia 15: 183 (1950). Kamiya, N. and Kuroda, K. XX. Ann. Meet. Bot. Soc. Jap. (1955) (unpublished). Kamiya, N. and Kuroda, K., Protoplasma 46: 423 (1956). Kuroda, K., Cytologia 21: 200 (1956). Linsbauer, K., Protoplasma 5 : 563 (1929). Sandan, T., Bot. Mag. Tokyo 68: 274 (1955). Valkanov, A., Protoplasma 20: 20 (1934).

\section{Anatomical Studies on the Vascular System of Mirabilis Jalapa L.}

\author{
by Riukiti INOUYE*
}

井上隆吉*：オシロイバナの維管束の解剖学的研究

Received in June 30, 1956

The peculiar feature of the vascular system in the stem of some nyctaginaceous plants and of the allied families has been recorded by many writers. It consists in the existence of the medullary bundles and in the thickening growth by the formation of the conjunctive tissue. According to de Bary (1877), in Mirabilis Jalapa and $M$. longiflora, only the separate and the fused leaf-trace bundles form the primary and medullary system; other bundles found external to it are produced by the extrafascicular cambium with their surrounding parenchyma cells, though they are apparently medullary, being imbedded in the tissue similar to the pith. Wilson (1924) has given a special remark on this point that in many species of the Amaranthaceae and Chenopodiaceae, the first-formed elements of the conjunctive tissue look like primary cells, so giving an appearance of the medullary bundles to those which are peripheral in reality. Contrary to de Bary, Maheshwari (1930), working on the ontogeny of the stem in Boerhaavia diffusa, has reported the presence of the primary bundles in the stelar periphery, and he has also insisted that the conjunctive tissue is not produced by the extrafascicular, but by the normal interfascicular cambium. The formation of the conjunctive tissue by the activity of the extrafascicular cambium which arises in the peircycle is, however, accepted by many

\footnotetext{
* Department of Biology, Saitama University, Urawa, Japan. 埼玉大学文理学部生物学教室
} 